\title{
66
}

\section{On the Distributed Fault Diagnosis of Computer Networks}

\author{
Henri NUSSBAUMER, Sailesh CHUTANI \\ Swiss Federal Institute of Technology, SWITZERLAND
}

We propose a general technique for the fault diagnosis of communication networks that is inspired by the theory of system-level diagnosis. This technique relies on the paradigm of comparison testing. A set of tasks, possibly implicit, is executed by the nodes in a network. The resulting agreements and disagreements in their results are used to diagnose all the faulty nodes and links with a high probability. The diagnosis algorithm proposed is applicable in a centralized as well as a distributed system. The accuracy of the diagnosis is controlled by the number of rounds of tasks performed. 